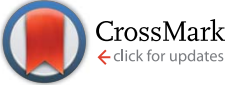

Cite this: RSC Adv., 2017, 7, 12835

\title{
Nanodiamonds as Raman probes for specifically targeted bioimaging: visualization and mechanism study of the biorecognition between nanodiamonds-EGF and EGFR $\uparrow$
}

\author{
Dandan Li, ${ }^{a b}$ Xin Chen, ${ }^{a b c}$ Hong Wang, ${ }^{c}$ Yuan Yu, ${ }^{a}$ Jie Liu, ${ }^{a}$ Yu Wang, ${ }^{d}$ Jinghua Zhang, ${ }^{d}$ \\ Meiling Zheng ${ }^{a}$ and Jinfang Zhi ${ }^{* a}$
}

\begin{abstract}
Nanodiamonds (NDs), with many superior properties, have been utilized as multifunctional drug delivery platforms, which are suitable for cancer cell targeting, imaging and therapeutic applications. In this paper, NDs were modified with epidermal growth factor (EGF) molecules through the amide bonds, and used as cancer cell seeking probes with confocal Raman microscopy to visualize the specific targeting recognition of EGF ligands and their receptors (EGFR) that were over-expressed on HeLa cells. Relying on the characteristic Raman signal of NDs at $1332 \mathrm{~cm}^{-1}$, Raman mapping was successfully utilized to reveal the specificity of EGF targeting ability under physiological conditions. Also, the specific recognition between EGF and its receptor was proven to be a dose-dependent process. Besides, the further influence of the specific recognition on HeLa cells such as the cell shape change and induced migration process was also investigated, and the related mechanism was discussed, showing that EGF recognition could change cell morphology and promote cancer cell migration, which might cause risks for the application of EGF molecules in targeting drug delivery systems. The present results provide a new perspective to evaluate and select suitable targeting molecules in future diagnostic and therapeutic applications.
\end{abstract}

Received 13th December 2016 Accepted 18th February 2017

DOI: 10.1039/c6ra28139g

rsc.li/rsc-advances

\section{Introduction}

Nanomaterials have emerged as powerful tools for enhancing drug delivery efficiency and biomedical imaging. ${ }^{1-3}$ With the higher porosity of the tumor vasculature and the lack of lymphatic drainage, the enhanced permeability and retention (EPR) effect permit nanoparticles to be more easily entrapped in most solid tumors. ${ }^{4}$ Among these promising systems, nanodiamonds (NDs) have been considered as attractive nanoparticles in biomedical applications because of their excellent chemical stability, superb biocompatibility and convenient surface modification properties. ${ }^{5-7}$ To date, various kinds of NDs-based optical probes to image biological systems have been demonstrated to be promising in biomedical imaging applications. ${ }^{8-16}$

Generally, as bio-probes, ND surfaces were usually modified or functionalized with targeting ligands to recognize biomolecules

${ }^{a}$ Laboratory of Photochemical Conversion and Optoelectronic Materials, Technical Institute of Physics and Chemistry, Chinese Academy of Sciences, Beijing 100190, PR China. E-mail: zhi-mail@mail.ipc.ac.cn; Fax: +86825 43537

${ }^{b}$ University of Chinese Academy of Sciences, Beijing 100049, PR China

${ }^{c}$ Department of Pharmaceutical Analysis, School of Pharmaceutical Sciences, Peking University, Beijing 100191, PR China

${ }^{d}$ Beijing Center for Physical and Chemical Analysis, Beijing 100089, PR China

$\dagger$ Electronic supplementary information (ESI) available. See DOI: 10.1039/c6ra28139g through specific interactions in biological systems. ${ }^{17-20}$ The surface homogenization and carboxylation could be realized through treatment with strong oxidizing acid to obtain carboxylated NDs (NDs-COOH), which can interact with biomolecules via covalent amide bonds. ${ }^{21}$ Some targeting ligands were successfully used to conjugate with NDs, demonstrating the capability of NDs as bio-probes for different applications. For examples, in a variety of NDs-based targeting drug delivery systems, NDs were not only utilized as simple drug carriers, but also as probes to locate the drug delivery systems. ${ }^{22-24}$ In another work, direct observation of the specific interaction between transferrin molecules and their receptors that were over-expressed on HeLa cells was successfully realized with fluorescent NDs as probes, which were conjugated with targeting ligands transferrin. ${ }^{25}$ Besides, Cheng et al. utilized Raman imaging technique based on the intrinsic Raman signal of NDs, instead of traditional fluorescent method, to observe the growth hormone receptors in one single A549 human lung epithelial cell, where growth hormone modified NDs were used as Raman probes. ${ }^{26}$ What's more, epidermal growth factor (EGF), as a stable targeting ligand with well-defined reaction sites for conjugation, can be used as a cancer seeking agent since its receptor (epidermal growth factor receptor, EGFR) is usually overexpressed in a variety of tumors such as gliomas and squamous carcinomas. ${ }^{27}$ It is thought that the EGF signal transduction is initiated by the EGFR dimerization, and upon the combination 
with EGF, the EGFR monomer in the membrane dimerizes and induces the intracellular activity. ${ }^{28}$ Significantly, it is worth noting that the specific biorecognition process between EGF and EGFR plays an crucial role in biological systems, ${ }^{29}$ which might interfere with the mRNA and protein expression in cancer cells, probably changing the tumor behaviors like aggression, migration and proliferation. ${ }^{\mathbf{3 0 , 3 1}}$ However, some of these influence were not clearly evaluated, which need special clarification and further investigation, like the expression level of some key mRNAs and proteins, cell activity affected and regulatory mechanism related. As such, NDs, since it could be used as probes to visualize the biorecognition process between EGF and EGFR, as well as to evaluate the further influence of this specific recognition on cancer cells and correlative mechanism, can serve as an excellent case for the investigation of this specific biorecognitions in a physiology condition.

For the visualization and mapping purpose, confocal Raman microscopy was used as imaging tool in this work. With recent development in spectroscopic techniques, Raman microscopy is effectively used in many biomedical studies because of its significant advantages over traditional fluorescent microscopy. For example, Raman imaging in general does not need cell labeling and fixation, which are usually essential in fluorescent imaging, providing facilities for the use of Raman microscopy in biomedical applications. ${ }^{\mathbf{1 6}}$ In addition, Raman imaging using biocompatible nanoparticles with intense and sharp Raman signal could avoid the autofluorescence in the sample and biobackground, which is quite a troublesome issue in fluorescence imaging. ${ }^{13}$ NDs, proven to be biocompatible nanoparticles, respond satisfactorily to the request mentioned above for its intrinsic and sharp Raman signal (located at $1332 \mathrm{~cm}^{-1}$ ). Hence, this intense Raman peak can be applied to locate NDs and the Raman imaging of nonfluorescent NDs probes in biological systems has been successfully accomplished, ${ }^{\mathbf{1 4}, 15}$ indicating the possibility of utilizing NDs as Raman probes to visualize the specific biorecognition process between EGF and EGFR.

In this study, we demonstrate the feasibility of using confocal Raman imaging to follow the specific targeting process of receptor-guided NDs probes on human cervical cancer cell line (HeLa), i.e., to present the distribution of EGF modified NDs and HeLa cells using Raman mapping. Furthermore, following influences of the biorecognition between EGF and EGFR on cell migration and related mechanisms were also specifically investigated here. The present results might propose a new perspective to evaluate the targeting molecules for future biomedical applications.

\section{Results and discussion}

\section{Characterization of NDs-EGF bioconjugate}

As described in the experimental section, the nanodiamonds (NDs) were carboxylated firstly, so as to obtain the $\mathrm{COOH}$ groups functional NDs, and then the EGF ligand was further assembled onto the NDs surface to obtain NDs-EGF bioconjugate. The successful modification of EGF on NDs was evaluated and the comparison of NDs and NDs-EGF on size distribution, zeta potential and FTIR spectra were displayed in the ESI. $\dagger$ As seen in Fig. S1A, $\uparrow$ after modification with EGF molecule, the particle size of NDs increased from $191.4 \mathrm{~nm}$ to $254.6 \mathrm{~nm}$, indicating the successful loading of EGF onto NDs. While in Fig. S1B, $\uparrow$ the zeta potential did not show obvious difference between NDs and NDs-EGF, and EGF modification minimally affected the particle zeta potential, suggesting that the interaction between NDs and EGF was more likely covalent bond, rather than electrostatic adherence. To validate this anticipation, we compared the FTIR spectra of NDs and NDs-EGF, as shown in Fig. S2. $\dagger$ The O-H bending near $1630 \mathrm{~cm}^{-1}$ and $\mathrm{C}=\mathrm{O}$ stretching at $1765 \mathrm{~cm}^{-1}$ are characteristic for the carboxylic group connected on the nanodiamonds surfaces. Characteristic protein amide peaks of EGF were observed at $1640 \mathrm{~cm}^{-1}(\mathrm{C}=\mathrm{O}$ stretching, amide $\mathrm{I})$ and 1540 $\mathrm{cm}^{-1}(\mathrm{~N}-\mathrm{H}$ bending, amide $\mathrm{II})$. When the EGF molecule was conjugated with NDs covalently, the $\mathrm{O}-\mathrm{H}$ disappeared and was replaced with amide peaks from EGF. Herein, the FTIR measurement ensured the covalent bond between NDs and EGF. Besides, confirmed with a BCA protein assay kit, the EGF conjugation efficiency was demonstrated to be $20.8 \pm 4.1 \mu \mathrm{g} / 100$ $\mu \mathrm{g}$ NDs in our experiments.

\section{Visualization of the interaction between NDs-EGF and HeLa cells}

The specific interaction between NDs-EGF and HeLa cells was investigated via confocal Raman mapping with NDs-EGF as Raman probes. Fig. 1 showed the Raman images of a HeLa cell incubated with NDs-EGF at the concentration of $25 \mu \mathrm{g} \mathrm{mL}{ }^{-1}$ for $4 \mathrm{~h}$, and following $48 \mathrm{~h}$ recovery. Basically, the distribution of NDs-EGF and HeLa cells in Fig. 1B were mapped out relying on the typical Raman spectra in Fig. 1C, where the sharp Raman peak at $1332 \mathrm{~cm}^{-1}$ represented the stretching vibrations of nanodiamonds in NDs-EGF conjugates, and the Raman bands in $2800-3200 \mathrm{~cm}^{-1}$ range could be assigned to the symmetric stretching vibration of $\mathrm{CH}_{2}$ and $\mathrm{CH}_{3}$ groups of the lipids in HeLa cells. ${ }^{16}$ The results demonstrated that the characteristic Raman peaks of NDs and HeLa cells were isolated and intense enough for Raman mapping.

Confocal Raman mapping provides the possibility to collect information of cNDs-EGF localization relative to the HeLa cells. Two-dimensional (2D) Raman spectral images were obtained by integrating the spatial distribution intensities of the selected Raman peaks which were assigned to the investigated the single cell and NDs-EGF. With dual-colored Raman imaging mode, Fig. 1B1 showed the outline of the HeLa cell in red color through the integrated Raman intensity of cell, and NDs-EGF distribution in green color (Fig. 1B2). Hence, the relative position of HeLa cell and NDs-EGF can be observed from the merged Raman images (Fig. 1B3). An important result was that a large amount of NDs-EGF (in yellow color) were located inside the cytoplasm of HeLa cells, without entering the nucleus in present incubation condition.

To ascertain the specific biorecognition between NDs-EGF and EGFR, a set of control experiments were conducted. Fig. 2 showed a comparison of the Raman images of HeLa cells respectively incubated with NDs and NDs-EGF to validate the 


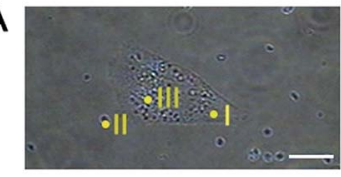

B

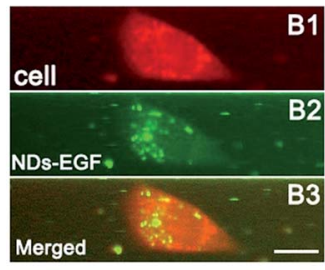

C

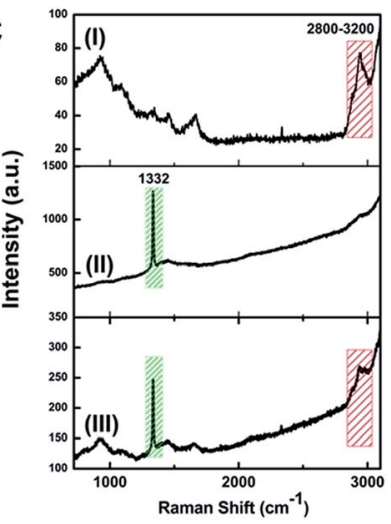

Fig. 1 Raman imaging of a HeLa cell incubated with NDs-EGF at the concentration of $25 \mu \mathrm{g} \mathrm{mL} \mathrm{L}^{-1}$ for $4 \mathrm{~h}$ and following $48 \mathrm{~h}$ recovery. (A) The bright field image of a HeLa cell. (B) The Raman images of the cell in (A). (B1) A Raman image of the cell, coming from the $\mathrm{C}-\mathrm{H}$ stretching vibrations of cells in the $2800-3200 \mathrm{~cm}^{-1}$ range, as displayed in spectrum (C) (I); (B2): a Raman image of NDs-EGF distribution in the cell in (A), coming from the nanodiamonds stretching vibrations at $1332 \mathrm{~cm}^{-1}$, as displayed in spectrum (C) (II); (B3): merged image of (B1) and (B2), with dual-colored Raman imaging mode. (C) The typical Raman spectra of the corresponding yellow spots in (A), and the spectrum III indicating the coexistence of NDs-EGF and cells (scale bar: $10 \mu \mathrm{m})$.
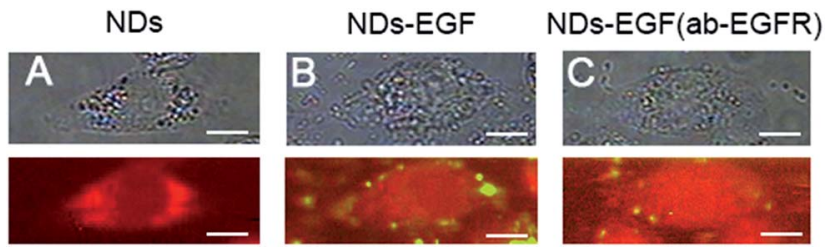

Fig. 2 Comparison of Raman images of HeLa cells incubated with NDs-EGF and NDs. (A) bare NDs (without EGF) treatment at the concentration of $25 \mu \mathrm{g} \mathrm{mL}^{-1}$ for $2 \mathrm{~h}$; (B) NDs-EGF conjugate treatment at the concentration of $25 \mu \mathrm{g} \mathrm{mL}^{-1}$ for $2 \mathrm{~h}$; and (C) ab-EGFR pretreated for $2 \mathrm{~h}$ followed by NDs-EGF bioconjugate treatment at the concentration of $25 \mu \mathrm{g} \mathrm{mL}^{-1}$ for $2 \mathrm{~h}$, respectively (scale bar: $10 \mu \mathrm{m}$ ).

specificity of EGF targeting ability. In Fig. 2, the bright field images of HeLa cells were shown above, and the Raman images of HeLa cells were shown below. Fig. 2A displayed the HeLa cells incubated with bare NDs (without EGF), Fig. 2B showed the HeLa cells treated with NDs-EGF bioconjugate, and Fig. 2C exhibited the HeLa cells pretreated with ab-EGFR for $2 \mathrm{~h}$ and then followed by NDs-EGF bioconjugate treatment.

As shown in Fig. 2A, when the HeLa cells were incubated with bare NDs (without EGF modification), the absent Raman signals of NDs suggested that the bare NDs were not bound to HeLa cells under this experiment condition, eliminating the interference of non-specific interaction coming from NDs, which was crucial in the observation of the specific recognition between EGF and EGFR. Whereas, as shown in Fig. 2B, an obvious NDs-EGF accumulation, like a yellow circle around the cell, could be observed when HeLa cells were incubated with NDs-EGF bioconjugate, confirming the specific biorecognition between NDsEGF and EGFR. In another control experiment, as shown in
Fig. 2C, when the HeLa cells were firstly pretreated with EGFR antibody (ab-EGFR) for $2 \mathrm{~h}$, i.e., the recognition sites were blocked and the interaction between EGF and its receptor EGFR was absent, and only a small amount of cNDs-EGF particles (yellow spots) accumulated on the cell, further assuring the specificity of the interaction between EGF ligands and their receptors on HeLa cells. Consequently, it is conceivable to conclude that the specific recognition or receptor-guided biorecognition between EGF and EGFR is attributed to the reaction between EGF (modified onto NDs) and EGFR (over-expressed on cancer cells), and through the specific recognition process, EGF modified NDs were easily delivered to the EGFR over-expressed cancer cells. Based on the intrinsic and sharp Raman signal of NDs probes, the biorecognition could be visualized through 2D Raman imaging.

Another issue regarding the dose-dependent behavior of the specific biorecognition between NDs-EGF and EGFR was evaluated through incubating HeLa cells with NDs-EGF bioconjugate in different concentration. As seen in Fig. 3, the bright field images of HeLa cells were shown in top part and the Raman images of HeLa cells were displayed in bottom. Fig. 3AC showed the HeLa cells incubated with NDs-EGF bioconjugate at the concentration of $25 \mu \mathrm{g} \mathrm{mL} \mathrm{m}^{-1}, 50 \mu \mathrm{g} \mathrm{mL} \mathrm{L}^{-1}$, and $100 \mu \mathrm{g}$ $\mathrm{mL}^{-1}$ for $2 \mathrm{~h}$, respectively. As the NDs-EGF concentration went up, the observed NDs-EGF conjugate accumulation on the cells increased, and more NDs-EGF signals were obtained in Fig. 3B and C, compared with that in Fig. 3A. Besides, in Fig. 3C, more NDs signals were observed around the cell, like a green circle, in comparison with the cell in Fig. 3B, where the distribution of NDs was still in green dots, showing the increased interaction between EGF ligands and their receptors, further revealing a dose-dependent behavior of the specific interaction between EGF ligands and their receptors on HeLa cells.

\section{NDs-EGF/EGFR biorecognition triggered cell migration process}

Interestingly, with prolonging incubation time, morphology change of HeLa cells was observed from the optical micrographs in Fig. 4, where the HeLa cells were incubated with NDs $\left(25 \mu \mathrm{g} \mathrm{mL} \mathrm{m}^{-1}\right)$ and NDs-EGF $\left(25 \mu \mathrm{g} \mathrm{mL}^{-1}\right)$ respectively for $4 \mathrm{~h}$ firstly, and after three times wash with PBS, the cells were incubated for an additional $48 \mathrm{~h}$ in a fresh media. As seen in Fig. 4, with NDs-EGF incubation, HeLa cells showed notable

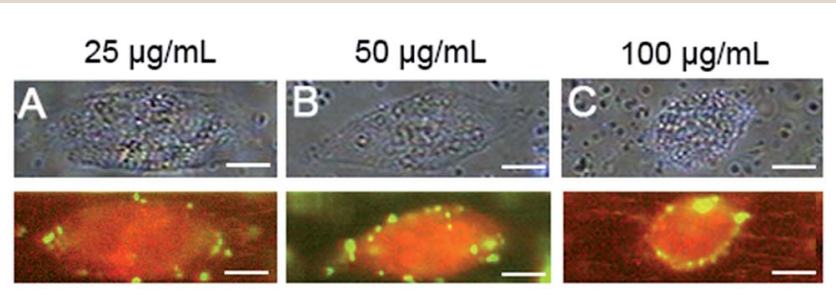

Fig. 3 Comparison of Raman images of HeLa cells incubated with NDs-EGF bioconjugate in different concentration: (A) $25 \mu \mathrm{g} \mathrm{mL}^{-1}$, (B) $50 \mu \mathrm{g} \mathrm{mL}^{-1}$, and (C) $100 \mu \mathrm{g} \mathrm{mL}-1$, for $2 \mathrm{~h}$ respectively (scale bar: $10 \mu \mathrm{m})$ 

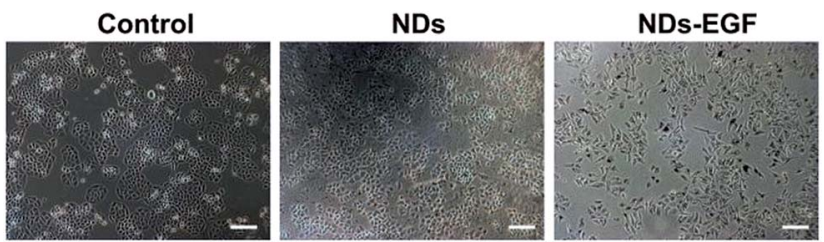

Fig. 4 The representative optical micrographs of the HeLa cells following the respective treatment of NDs and NDs-EGF, with DMEM incubation as control (scale bar: $100 \mu \mathrm{m}$ ).

change in cell morphology and relative lack of cell adhesion in comparison with the control group and NDs treated group.

To further investigate the performance of HeLa cell shape change, Raman imaging was used to reveal the details. Fig. 5 displayed the representative Raman images of single HeLa cells incubated with NDs or NDs-EGF. For the cells treated with NDsEGF bioconjugate (Fig. 5A), the morphology of the cells changed into an elongated shape, from epithelial-like to mesenchymallike. However, when treated with NDs, HeLa cells remained unchanged as the epithelial-like shape, revealing that NDs itself did not lead to the changes in cell morphology (Fig. 5B). The dramatic changes in cell morphology induced by NDs-EGF also suggested that the HeLa cells extended the leading edge and retracted their trailing edge (Fig. 5A5), indicating the possibility of cell migration.

Cell migration is an important step in tumor cell growth, invasion and metastasis. ${ }^{32}$ Hence, the influence of NDs-EGF bioconjugate on cell migration needs further evaluation. The cell migration was characterized with transwell migration assays. The migration ability of HeLa cells that were respectively treated with bare NDs, NDs-EGF and EGF (at the concentration equivalent to that of NDs-EGF) was fully investigated, using the

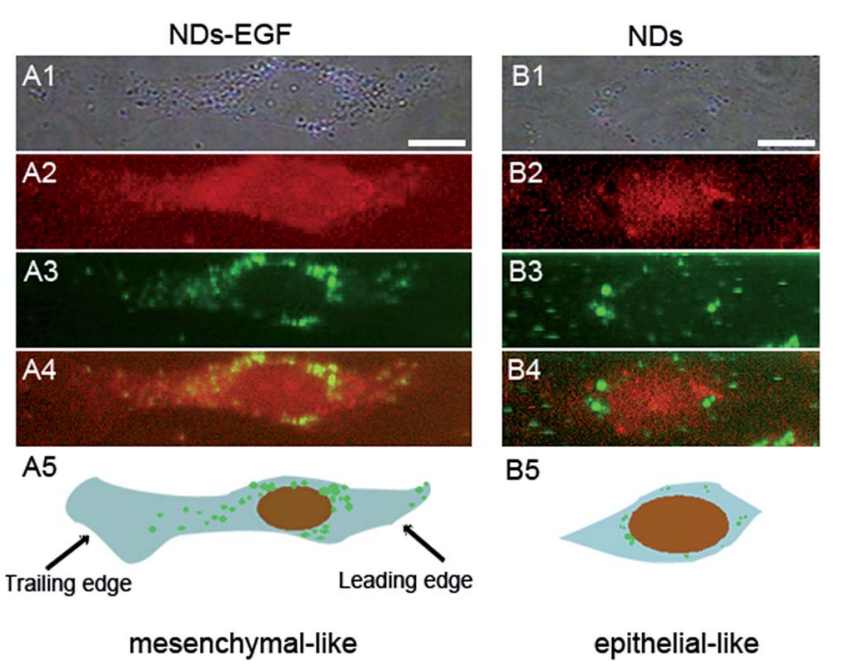

Fig. 5 Comparison of the morphology of HeLa cells treated with (A) NDs-EGF bioconjugate and (B) NDs at the concentration of $25 \mu \mathrm{g}$ $\mathrm{mL}^{-1}$ for $4 \mathrm{~h}$, with another $48 \mathrm{~h}$ incubation for recovery. (A1 and B1) The bright field images of HeLa cells. (A2 and B2) The Raman images of the cells. (A3 and B3) The Raman images of NDs-EGF/NDs distribution in the cell. (A4 and B4) Merged images of cells and NDs-EGF. (A5 and B5) The illustration of cell morphology change (scale bar: $10 \mu \mathrm{m}$ ).
A

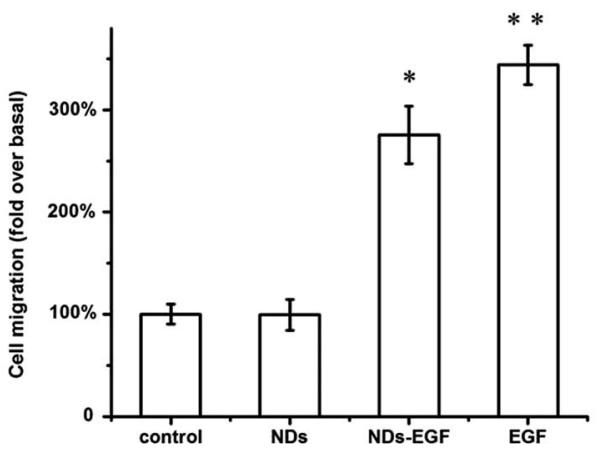

B
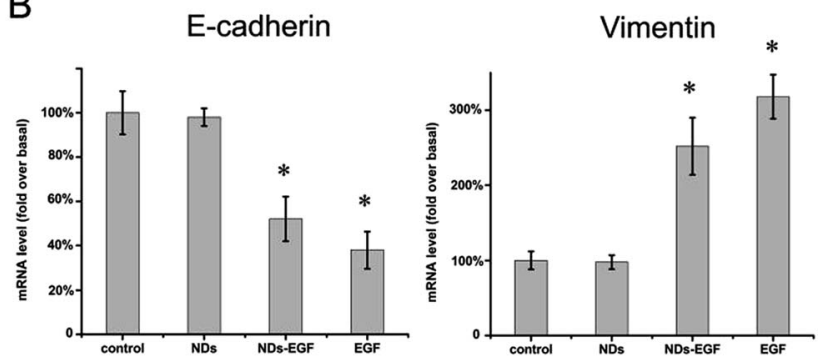

Fig. 6 Influence on HeLa cell migration of NDs-EGF treatment. (A) Quantification of the migration rates of HeLa cells following the treatments of NDs, NDs-EGF and EGF, with DMEM incubation as control, revealing the enhanced cell migration ability induced by NDsEGF; (B) evaluation of the mRNA expression of HeLa cells treated with NDs, NDs-EGF and EGF, with DMEM incubation as control, showing the $E$-cadherin down-regulation and vimentin up-regulation induced by NDs-EGF. The rates are normalized to controls. Error bars represent \pm SD. $* p<0.05 . * * p<0.01$

untreated HeLa cells as control group, and the results were shown in Fig. 6. As shown in Fig. 6A, compared with the control group, no obvious migration difference occurred for the cells treated with NDs, while NDs-EGF and EGF treatment could significantly induce migration of HeLa cells, which is based on the disruption of cell-cell contact by EGF molecules. ${ }^{31}$ And the related mechanism of cell migration induced by NDs-EGF was discussed in the next part.

Besides, because of the migration induced by NDs-EGF, we should focus on another related issue, cell cytotoxicity of NDsEGF. The cytotoxicity assessment of NDs and NDs-EGF was conducted in HeLa cells with CCK-8 assays, as displayed in Fig. S3 in the ESI. $\dagger$ Fig. S3 $\uparrow$ showed no significant difference in cell viability between negative control (only DMEM), NDs and NDs-EGF, indicating good biocompatibility of NDs and NDsEGF.

As mentioned above, the obvious morphological changes, from epithelial-like to mesenchymal-like could be observed when HeLa cells were incubated with NDs-EGF (Fig. 5A5). In addition, the cell migration induced by NDs-EGF bioconjugate was also confirmed. Whereas, the related mechanism of cell morphology change as well as migration ability induced by NDsEGF was still unclear.

The main aim of this part is to make further investigation into the migration mechanism induced by NDs-EGF. Cell migration regulated by EGF-mediated signaling pathway was 
reported before, where E-cadherin played a crucial role in this pathway. ${ }^{33}$ E-Cadherin is a calcium-dependent cell-cell adhesion molecule with high expression level in epithelial cells. ${ }^{34}$ The E-cadherin expression levels in HeLa cells, which were treated with NDs, NDs-EGF and EGF respectively, were evaluated and the result was shown in Fig. 6B. It was observed that, compared with NDs, NDs-EGF bioconjugate and EGF molecules could significantly decrease E-cadherin mRNA expression in treated cells, suggesting the NDs-EGF induced E-cadherin down-regulation in HeLa cells.

As a well-known hallmark of epithelial-mesenchymal transition (EMT), down-regulation of E-cadherin raised the possibility of EMT in HeLa cells. Therefore, we further investigated whether NDs-EGF could induce EMT through evaluating the expression level of a key mesenchymal marker, vimentin. ${ }^{35}$ As shown in Fig. 6B, NDs-EGF and EGF molecules could obviously increase the mRNA expression of vimentin, indicating the possibility of EMT in HeLa cells via up-regulation of vimentin induced by NDs-EGF. Therefore, we verified that NDs-EGF could induce E-cadherin down-regulation and vimentin up-regulation through an EMT-dependent mechanism in HeLa cells. This outcome further indicated that EGF molecules might cause EMT and migration risks for cancer cells when it is used as targeting ligand for specific anticancer drug delivery, which emphasized the importance of targeting molecule selection in further therapeutic applications.

Moreover, compared with that of the EGF free group, an slight migration inhibition effect, approximately $20-30 \%$ as displayed in Fig. 6A, can be observed when the HeLa cells were treated with NDs-EGF, and similar conclusion was obtained for the mRNA expression level of the E-cadherin and vimentin (Fig. 6B). This result indicated that NDs, as carriers, could probably interfere with receptor binding or serve as receptor inhibitors. Because the affinity of NDs-EGF to EGFR mainly depends on the native conformation, after conjugating EGF molecules to NDs, the occupation of EGF onto cell surface may be reduced, resulting in a loss of receptor-binding activity of EGF. However, more work are still necessary if we want to fully understand the related processes. Besides, the study and evaluation of the further influence of NDs-EGF bioconjugate on cell endocytosis, metastasis and some other aspects will continue.

\section{Experimental}

\section{Materials}

Nanodiamonds (NDs), with $100 \mathrm{~nm}$ original sizes, were purchased from Guangzhou Kesiman Co., Ltd. (China). Hematein staining solution, $N$-hydroxysuccinimide (NHS) and 1-(3dimethylaminopropyl)-3-ethylcarbodiimide hydrochloride (EDC) were provided by Sigma-Aldrich (St. Louis, MO, USA), which were used to active the interaction of NDs with human epidermal growth factor (EGF). BCA protein assay kit, from Pierce Biotechnology (Rockford, USA), was utilized to calculate the amount of EGF molecules. The antibody described in this study was anti-EGFR (Santa Cruz Biotechnology, USA), for EGFR blocking experiments. Dulbecco's modified Eagle's medium (DMEM) and modified Dulbecco's phosphate buffered saline
(PBS) were purchased from Hyclone (Thermo Scientific, China). Penicillin-streptomycin and $0.25 \%$ trypsin-EDTA solution were provided by Hyclone (Thermo Scientific, USA), and fetal bovine serum (FBS) was bought from Hyclone (Thermo Scientific, New Zealand). Cell Counting Kit-8 (CCK-8) was purchased from Dojindo Co., Ltd (Tokyo, Japan) to evaluate the cell viability. For the cell migration investigation, transwell assay kit $8 \mathrm{~mm}$ pores was from FALCON (BD Biosciences, USA). In total RNA extraction and real-time quantitative polymerase chain reaction analysis experiments, RNeasy ${ }^{\circledR}$ plus mini kits and Omniscript ${ }^{\circledR}$ reverse transcription kits were bought from QIAGEN GmbH (Hilden, Germany) and 2X Power SYBR Green PCR Master Mix was purchased from Applied Biosystems (Thermo Scientific, China). Other reagents were bought from China National Medicine Corporation (Shanghai, China) unless indicated otherwise. All the chemicals were reagent grade and used without further purification.

\section{Formation and characterization of NDs-EGF bioconjugate}

Nanodiamonds (NDs) were firstly surface-functionalized to form carboxylated NDs by treatment with strong oxidizing acid. ${ }^{36}$ NDs were first immersed in a $3: 1(\mathrm{v} / \mathrm{v})$ mixture of concentrated $\mathrm{H}_{2} \mathrm{SO}_{4}$ and $\mathrm{HNO}_{3}$ at $70{ }^{\circ} \mathrm{C}$ for $24 \mathrm{~h}$, then treated with $\mathrm{NaOH}$ aqueous solution $(0.1 \mathrm{M})$ at $70{ }^{\circ} \mathrm{C}$ for $1 \mathrm{~h}$, and finally immersed in $\mathrm{HCl}$ aqueous solution $(0.1 \mathrm{M})$ at $70{ }^{\circ} \mathrm{C}$ for $1 \mathrm{~h}$. The treated NDs were subsequently washed with distilled water for several times and the sediments was dried in air to obtain the carboxylated NDs. For formation of NDs-EGF bioconjugate, NDs $\left(1 \mathrm{mg} \mathrm{mL} \mathrm{m}^{-1}, 100\right.$ $\mu \mathrm{L})$ were incubated with $\mathrm{EGF}\left(1 \mathrm{mg} \mathrm{mL}^{-1}, 100 \mu \mathrm{L}\right)$ in a thermomixer at $37{ }^{\circ} \mathrm{C}$ for $1 \mathrm{~h}$, with the addition of NHS $\left(2 \mathrm{mg} \mathrm{mL}{ }^{-1}\right.$, $100 \mu \mathrm{L})$ and EDC $\left(2 \mathrm{mg} \mathrm{mL}{ }^{-1}, 100 \mu \mathrm{L}\right)$ to activate the reaction. Then the NDs-EGF bioconjugate produced were purified through centrifugation for three times and resuspended in DMEM for immediate use. Fourier Transform Infrared (FTIR) Spectroscopy (Excalibur 3100, Varian, America) was used to ensure the covalent bond between NDs and EGF. The size distribution and zeta potentials of prepared NDs-EGF bioconjugate were recorded in distilled water with a zeta-potential analyzer, Zetasizer 3000HS (Malvern Instruments, England). The conjugation efficiency of EGF in NDs-EGF were determined using a Pierce ${ }^{8}$ BCA protein assay kit (normalized per $100 \mu \mathrm{g}$ NDs), through calculating the amounts of EGF in the supernatant of the reaction solution before and after NDs-EGF bioconjugate formation.

\section{Visualization of the interaction between NDs-EGF and HeLa cell}

Nanodiamonds as Raman probes to visualize the interaction between NDs-EGF and HeLa cells was performed using a confocal Raman microscope (RAMAN-11, Nanophoton, Osaka, Japan) with $532 \mathrm{~nm}$ laser wavelength under the slit-scanning mode. The laser intensity used at the sample plane was $0.7 \mathrm{~mW} \mu \mathrm{m}^{-2}$. The exposure time for each line was $5 \mathrm{~s}$ and the acquisition time for each sample was 5-8 min. A 60X/1.2 NA water immersion objective lens (UPLSAPO, Olympus) was used in the microscope stage. The spectral resolution was $3 \mathrm{~cm}^{-1}$. The spatial resolution: the pixel size was $431.5 \mathrm{~nm}$. To observe the specific recognition 
process between EGF and its receptor, HeLa cells were treated with NDs-EGF bioconjugate $\left(25 \mu \mathrm{g} \mathrm{mL}{ }^{-1}\right)$ at $37{ }^{\circ} \mathrm{C}$ for $2 \mathrm{~h}$ firstly, and then washed with PBS for three times. The treated live cells in PBS were placed at the microscope stage directly. Two control experiments were carried out to confirm that the specific biorecognition of EGF and EGFR on HeLa cells occurs exclusively. One control is that the HeLa cells were firstly treated with NDs (25 $\mu \mathrm{g} \mathrm{mL}{ }^{-1}$ ) and then incubated at $37^{\circ} \mathrm{C}$ for $2 \mathrm{~h}$, and the other is that the HeLa cells were firstly blocked with anti-EGFR $(25 \mu \mathrm{g}$ $\mathrm{mL}^{-1}$ ) and then incubated at $37^{\circ} \mathrm{C}$ for $2 \mathrm{~h}$ before treatment with NDs-EGF bioconjugate. In addition, for the dose-dependent behavior of the specific recognition process, HeLa cells were treated with NDs-EGF bioconjugate at the concentration of $25 \mu \mathrm{g}$ $\mathrm{mL}^{-1}, 50 \mu \mathrm{g} \mathrm{mL} \mathrm{m}^{-1}$ and $100 \mu \mathrm{g} \mathrm{mL} \mathrm{m}^{-1}$ separately at $37^{\circ} \mathrm{C}$ for $2 \mathrm{~h}$ firstly, and then washed with PBS for three times before Raman imaging. To ensure the reliability of Raman imaging and quantitative analysis, the experiments were repeated for several times $(N>5)$ and 8-10 cells were investigated each time.

\section{HeLa cell migration assay}

Cell migration was measured using a transwell kit with $8 \mathrm{~mm}$ pores as previously described. ${ }^{33}$ HeLa cells were suspended in serum-free DMEM medium containing NDs $\left(25 \mu \mathrm{g} \mathrm{mL}{ }^{-1}\right)$, NDsEGF $\left(25 \mu \mathrm{g} \mathrm{mL}^{-1}\right.$ ) and EGF (at the concentration equivalent to that of NDs-EGF) respectively, and plated onto the transwell insert placed in the chamber containing DMEM with $10 \%$ FBS. The chambers were incubated at $37{ }^{\circ} \mathrm{C}$ in $5 \% \mathrm{CO}_{2}$ for $16 \mathrm{~h}$. Migrated cells, which adhered to the lower surface of the porous membrane, were fixed in $4 \%$ paraformaldehyde for $15 \mathrm{~min}$, and then stained with hematein staining solution. Unmigrated cells on the upper membrane surface were removed with a cotton swab. The migration was quantified by analyzing at least six random fields per filter for each independent experiment. All experiments were repeated in triplicate.

\section{HeLa cell viability assay}

HeLa cells were first seeded in a 96-well plate $\left(1 \times 10^{4}\right.$ cells per well) in Dulbecco's modified Eagle's medium (DMEM) with $10 \%$ fetal bovine serum and antibiotics, and the cells were grown to $50-60 \%$ confluency. The media was aspirated and the cells were washed three times with PBS. HeLa cells were incubated with fresh media containing NDs $\left(25 \mu \mathrm{g} \mathrm{mL}{ }^{-1}\right)$ and NDs-EGF $(25 \mu \mathrm{g}$ $\mathrm{mL}^{-1}$ ) for $4 \mathrm{~h}$, respectively. After three times wash with PBS, HeLa cells were incubated for an additional $48 \mathrm{~h}$ in a fresh media. Control experiments were conducted with HeLa cells in triplicate. Cell viability was measured by CCK- 8 assay with $450 \mathrm{~nm}$ wavelength. Three parallel experiments were performed.

\section{Investigation of the cell migration mechanism}

The migration mechanism of HeLa cell treated with NDs-EGF was evaluated with the previously reported method, ${ }^{33}$ through total RNA extraction and real-time quantitative polymerase chain reaction (qPCR) analysis. Total RNA from pancreas was extracted with the RNeasy ${ }^{\circledR}$ plus mini kit. With Omniscript ${ }^{\circledR}$ reverse transcription kit, cDNA was prepared by in vitro transcription, which was used as a template for PCR amplification in a $20 \mu \mathrm{L}$ reaction volume using gene-specific primer pairs. 2X Power SYBR Green PCR Master Mix was used. For the amplification, the following cycle was used: $95^{\circ} \mathrm{C}$ for $10 \mathrm{~min}$, followed by 40 cycles of denaturation at $95^{\circ} \mathrm{C}$ for $15 \mathrm{~s}$ and primer extension at $60^{\circ} \mathrm{C}$ for $1 \mathrm{~min}$. The comparative $2^{-\Delta \Delta C_{t}}$ method was utilized to calculate the relative changes in gene expression, with $18 \mathrm{~s}$ for normalization. Primer sets for vimentin, E-cadherin and 18s: vimentin F, AAGGCGAGGAGAGCAGGATT, R, GGTCATCGTGATGCTGAGAAG; E-cadherin F, CCTGGCAAGATGCAGAAACTG, R, TGCTGCTTGGCCTCAAAAT; 18s F, GTAACCCGTTGAACCCCATT, R, CCATCCAATCGGTAGTAGCG. For each reaction, a parallel reaction that lacked template was performed as a negative control. All RT-PCR reactions for each sample were performed in triplicate.

\section{Statistical analysis}

Data were expressed as mean \pm standard (SD) and were analyzed by Student's $t$-test when appropriate. $p<0.05$ was considered statistically significant.

\section{Conclusions}

In summary, we present the direct observation of the specific targeting recognition of EGF and its receptor (EGFR), which is overexpressed on HeLa cell. And the visualization was realized with NDs as Raman probes by confocal Raman microscopy relying on the intrinsic Raman signal of NDs at $1332 \mathrm{~cm}^{-1}$. Besides, the dose-dependent behavior of the specific recognition between EGF and EGFR was also demonstrated. In addition, after NDs-EGF treatment, an obvious morphological change and enhanced motility of HeLa cells were observed, and the EMT dependent migration process was also characterized with the mRNA expression level of the down-regulated Ecadherin and up-regulated vimentin.

Finally, the present study highlights the application of NDs, which could be used as Raman imaging probes for cellular observation of specific recognition process in a physiology condition, combined with the further investigation of the influence on HeLa cells after recognition, providing a new perspectives for the evaluation and selection of suitable targeting molecules in potential drug delivery applications.

\section{Acknowledgements}

The authors appreciate the supports of the International Science \& Technology Cooperation Program of China (Grant No. 2013DFG50150), the Natural Foundation of Sciences of the People's Republic of China (Grant No. 21375137). Also, this work is supported by the Open Research Fund of State Key Laboratory of Bioelectronics, Southeast University.

\section{Notes and references}

1 W. H. De Jong and P. J. Borm, Int. J. Nanomed., 2008, 3, 133149.

2 S. J. Rosenthal, J. C. Chang, O. Kovtun, J. R. McBride and I. D. Tomlinson, Chem. Biol., 2011, 18, 10-24. 
3 D. T. Nguyen and K.-S. Kim, Korean J. Chem. Eng., 2014, 31, 1289-1305.

4 C.-L. Tseng, T.-W. Wang, G.-C. Dong, S. Yueh-Hsiu Wu, T.-H. Young, M.-J. Shieh, P.-J. Lou and F.-H. Lin, Biomaterials, 2007, 28, 3996-4005.

5 V. Vairakkannu, T. Yan-Kai, C. Huan-Cheng and L. ChungLeung, Nanotechnology, 2009, 20, 425103.

6 A. M. Schrand, H. Huang, C. Carlson, J. J. Schlager, E. Ōsawa, S. M. Hussain and L. Dai, J. Phys. Chem. B, 2007, 111, 2-7.

7 A. M. Schrand, S. A. C. Hens and O. A. Shenderova, Crit. Rev. Solid State Mater. Sci., 2009, 34, 18-74.

8 A. M. Schrand, J. B. Lin, S. C. Hens and S. M. Hussain, Nanoscale, 2011, 3, 435-445.

9 B.-M. Chang, H.-H. Lin, L.-J. Su, W.-D. Lin, R.-J. Lin, Y.-K. Tzeng, R. T. Lee, Y. C. Lee, A. L. Yu and H.-C. Chang, Adv. Funct. Mater., 2013, 23, 5737-5745.

10 Y. Fu, N. An, S. Zheng, A. Liang and Y. Li, Diamond Relat. Mater., 2012, 21, 73-76.

11 Y.-q. Li and X.-p. Zhou, Diamond Relat. Mater., 2010, 19, 1163-1167.

12 V. N. Mochalin, O. Shenderova, D. Ho and Y. Gogotsi, Nat. Nanotechnol., 2012, 7, 11-23.

13 J.-I. Chao, E. Perevedentseva, P.-H. Chung, K.-K. Liu, C.-Y. Cheng, C.-C. Chang and C.-L. Cheng, Biophys. J., 2007, 93, 2199-2208.

14 P. Elena, C. Chih-Yuan, C. Pei-Hua, T. Jhih-Sian, H. Yu-Hsin and C. Chia-Liang, Nanotechnology, 2007, 18, 315102.

15 E. Perevedentseva, Y.-C. Lin, M. Jani and C.-L. Cheng, Nanomedicine, 2013, 8, 2041-2060.

16 E. Perevedentseva, S. F. Hong, K. J. Huang, I. T. Chiang, C. Y. Lee, Y. T. Tseng and C. L. Cheng, J. Nanopart. Res., 2013, 15, 1-12.

17 T. S. Huang, Y. Tzeng, Y. K. Liu, Y. C. Chen, K. R. Walker, R. Guntupalli and C. Liu, Diamond Relat. Mater., 2004, 13, 1098-1102.

18 D. T. Tran, V. Vermeeren, L. Grieten, S. Wenmackers, P. Wagner, J. Pollet, K. P. F. Janssen, L. Michiels and J. Lammertyn, Biosens. Bioelectron., 2011, 26, 2987-2993.
19 A. Krueger, J. Stegk, Y. Liang, L. Lu and G. Jarre, Langmuir, 2008, 24, 4200-4204.

20 L. Marcon, M. Wang, Y. Coffinier, F. Le Normand, O. Melnyk, R. Boukherroub and S. Szunerits, Langmuir, 2010, 26, 10751080.

21 H.-D. Wang, Q. Yang and C. H. Niu, Diamond Relat. Mater., 2010, 19, 441-444.

22 Z. Wang, Z. Tian, Y. Dong, L. Li, L. Tian, Y. Li and B. Yang, Diamond Relat. Mater., 2015, 58, 84-93.

23 E. K.-H. Chow and D. Ho, Sci. Transl. Med., 2013, 5, 216 rv214.

24 X.-Q. Zhang, R. Lam, X. Xu, E. K. Chow, H.-J. Kim and D. Ho, Adv. Mater., 2011, 23, 4770-4775.

25 M.-F. Weng, S.-Y. Chiang, N.-S. Wang and H. Niu, Diamond Relat. Mater., 2009, 18, 587-591.

26 C.-Y. Cheng, E. Perevedentseva, J.-S. Tu, P.-H. Chung, C.-L. Cheng, K.-K. Liu, J.-I. Chao, P.-H. Chen and C.-C. Chang, Appl. Phys. Lett., 2007, 90, 163903.

27 V. P. Collins, Glia, 1995, 15, 289-296.

28 L. J. Pike, Biochim. Biophys. Acta, Mol. Cell Res., 2005, 1746, 260-273.

29 A. A. Bhirde, V. Patel, J. Gavard, G. Zhang, A. A. Sousa, A. Masedunskas, R. D. Leapman, R. Weigert, J. S. Gutkind and J. F. Rusling, ACS Nano, 2009, 3, 307-316.

30 E. Tashiro, S. Henmi, H. Odake, S. Ino and M. Imoto, Biochem. Biophys. Res. Commun., 2016, 477, 801-806.

31 X. Chen, D. Li, H. Wang, Y.-y. Jiao, H. Wang, Y. Yu and J. Zhi, RSC Adv., 2016, 6, 44543-44551.

32 D. A. Lauffenburger and A. F. Horwitz, Cell, 1996, 84, 359369.

33 S. Magi, Y. Saeki, M. Kasamatsu, E. Tashiro and M. Imoto, PLoS One, 2014, 9, 7.

34 A. Nagafuchi, Y. Shirayoshi, K. Okazaki, K. Yasuda and M. Takeichi, Nature, 1987, 329, 341-343.

35 R. P. Singh, A. Tyagi, G. Sharma, S. Mohan and R. Agarwal, Clin. Cancer Res., 2008, 14, 300-308.

36 X. Chen, H. Wang, D. Li, Y. Yu and J. Zhi, Phys. Status Solidi A, 2016, 213, 2131-2137. 